\title{
Sex-specific genetic determinants for arterial stiffness in Dahl salt-sensitive hypertensive rats
}

\author{
Julius L. Decano, Khristine A. Pasion, Nicole Black, Nicholas J. Giordano, Victoria L. Herrera and Nelson Ruiz-Opazo*
}

\begin{abstract}
Background: Arterial stiffness is an independent predictor of cardiovascular outcomes in hypertensive patients including myocardial infarction, fatal stroke, cerebral micro-bleeds which predicts cerebral hemorrhage in hypertensive patients, as well as progression to hypertension in non-hypertensive subjects. The association between arterial stiffness and various cardiovascular outcomes (coronary heart disease, stroke) remains after adjusting for age, sex, blood pressure, body mass index and other known predictors of cardiovascular disease, suggesting that arterial stiffness, measured via carotid-femoral pulse wave velocity, has a better predictive value than each of these factors. Recent evidence shows that arterial stiffening precedes the onset of high blood pressure; however their molecular genetic relationship (s) and sex-specific determinants remain uncertain. We investigated whether distinct or shared genetic determinants might underlie susceptibility to arterial stiffening in male and female Dahl salt-sensitive rats. Thus, we performed a genome-wide scan for quantitative trait loci (QTLS) affecting arterial stiffness in six-week old F2 (Dahl S x R)-intercross male and female rats characterized for abdominal aortic pulse wave velocity and aortic strain by high-resolution ultrasonography.

Results: We detected five highly significant QTLs affecting aortic stiffness: two interacting QTLS (AS-m1 on chromosome 4 and AS-m2 on chromosome16, LOD 8.8) in males and two distinct interacting QTLs (AS-f1 on chromosome 9 and AS-f2 on chromosome11, LOD 8.9) in females affecting pulse wave velocity. One QTL (AS-1 on chromosome 3, LOD 4.3) was found to influence aortic strain in a sex-independent manner. None of these arterial stiffness QTLs co-localized with previously reported blood pressure QTLs detected in equivalent genetic intercrosses.

Conclusions: These data reveal sex-specific genetic determinants for aortic pulse wave velocity and suggest distinct polygenic susceptibility for arterial stiffness and salt-sensitive hypertension in Dahl rats based upon reported blood pressure QTLs in equivalent (Dahl S X R)-intercrosses.
\end{abstract}

Keywords: Genetic linkage, QTL, Arterial stiffness, Pulse wave velocity, Arterial strain, Dahl rat

\section{Background}

Several studies support a genetic component contributing to arterial stiffness. Heritability for pulse wave velocity (PWV) in young adults in the Georgia Cardiovascular Twin Study was about 0.43 and 0.53 for aorto-radial and aortodorsalis-pedis PWV respectively [1]. In the Framingham Heart Study cohort a heritability of 0.4 was reported [2]. Genetic linkage studies in the Framingham cohort reported several regions of linkage, albeit suggestive rather than significant, for carotid femoral PWV on chromosomes 2, 7 ,

\footnotetext{
* Correspondence: nruizo@bu.edu

Department of Medicine, Whitaker Cardiovascular Institute, Boston University School of Medicine, 700 Albany Street, W-609, Boston, MA 02118, USA
}

13 and 15 (LOD scores > 2.0) [2]. Analysis of common polymorphisms in several candidate genes have been performed, however only CYP11B2 and NOS3 have been confirmed by genome-wide linkage studies [3] as being associated with arterial stiffness. While these observations demonstrate the heritability and the quantitative trait nature of arterial stiffness, they also illustrate the complexity of arterial stiffness, and hence the ensuing difficulties in the study of its genetic basis in the general population. These suggest the need for systematic animal model studies in dissecting causal paradigms for susceptibility.

To date, however, no genome wide studies of arterial stiffness in the context of polygenic hypertension have been done in animal models. Gaugier and collaborators 
identified a QTL controlling elastin content in rat aorta [4]; however, their study was done in non-hypertensive rat strains: Brown Norway rat strain and the LOU rat strain. More specifically, the Brown Norway rat strain shows an aortic elastin deficit by $5.8 \%$ by dry-weight compared with the inbred LOU rat, and is also highly susceptible to spontaneous rupture of the internal elastic lamina in its abdominal aorta - however Brown Norway rats are not hypertensive. Additionally, analysis of the aortic elastic network and related phenotypes in seven inbred rat strains was reported; however, these were all done in non-hypertensive rat strains [5].

Given that arterial stiffness precedes hypertension in a Dahl salt-sensitive hypertensive stroke-prone rat model [6] as it does in humans; this experimental model system allows the systematic dissection as to whether common or distinct genetic determinants underlie hypertension and arterial stiffness. Having performed QTL analyses for blood pressure in male and female F2 ([Dahl S] Dahl salt-sensitive $x$ [Dahl $R$ ] Dahl salt-resistant)-intercrosses $[7,8]$ we performed a total genome scan for QTLs affecting aortic Pulse Wave Velocity (PWV) and aortic Strain as quantitative measures of arterial stiffness using an F2 (Dahl S x R)-intercross male and female rat populations phenotyped for aortic PWV and aortic Strain at six weeks of age prior to the onset of hypertension. Results were then compared to blood pressure QTLs detected in previous genome scans performed in male [7] and female F2 [Dahl S x R]-intercross rats $[7,8]$. Data demonstrate that distinct QTLs underlie male and female arterial stiffness in Dahl rats. Moreover, none of the arterial stiffness QTLs co-localized with blood pressure QTLs detected in genetically equivalent F2 [Dahl S x R]-intercrosses indicating distinct genetic mechanisms underlying arterial stiffness and hypertension in Dahl rats.

\section{Results and discussion Study cohorts}

To investigate the genetic determinants that contribute to arterial stiffness we performed a genome scan for QTLs affecting aortic (between superior mesenteric artery and left renal artery) PWV and strain in an F2 (Dahl S x R)intercross male and female rat populations phenotyped for aortic PWV and aortic strain by high-resolution ultrasonography. We implemented the following experimental design: F2 hybrids were produced from dams on Purina $5001(0.4 \% \mathrm{NaCl})$ during gestation and lactating and maintained on Purina 5001 at weaning. Ultrasound measurements of abdominal aortic PWV and strain were done at 6 weeks of age following identical procedures. Rather than carotid-femoral PWV measurements, we measured aortic PWV between two points: below the superior mesenteric artery and above the left renal artery. This allows reproducible measurements of aortic PWV, between two points of the same artery guided by clear anatomic landmarks. We measured aortic PWV and aortic Strain in 142 male and 140 female subjects. The cohort was genotyped with 110 informative markers for our (Dahl S x R) intercross with an average density of $21.7 \mathrm{Mbp}$ distance between two markers.

In order to determine the earliest developmental age for arterial stiffness measurements and potentially eliminate blood pressure as a confounder in our ultrasound measurements we first analyzed the time course of development of arterial stiffness in parental Dahl S and Dahl $\mathrm{R}$ rats. As shown in Fig. 1 aortic PWV was significantly elevated in Dahl S male (Fig. 1a, $P<0.001$ ) and Dahl $\mathrm{S}$ female (Fig. 1b, $P<0.00001$ ) subjects when compared with corresponding Dahl R subjects at 6 weeks of age. The fact that the phenotypic differences in arterial stiffness measures are detected at 6 weeks of age in animals maintained on a $0.4 \% \mathrm{NaCl}$ regular rat chow diet, prior to the onset of high blood pressure, eliminates hypertension as a potential confounder in our genetic linkage study. The mean aortic PWV values in both male and female F2 populations fall between the parental mean values (Table 1) suggesting a co-dominant effect for both Dahl S and Dahl R alleles on the trait. In contrast, the mean aortic strain values for the F2 male and female cohorts approached the value obtained for the Dahl R rats (Table 1) indicating a dominance effect of the alleles for the trait (recessive for Dahl S and dominant for Dahl $\mathrm{R}$ respectively). This differential trend also indicates that aortic PWV is a distinct trait from aortic strain.

\section{Total genome scan analysis}

We next performed a total genome scan for QTLs affecting aortic PWV using 6 weeks-old F2 (Dahl S x R)-intercross male and female rats. Marker regression analysis did not detect any significant PWV QTL in both male and female cohorts, only suggestive QTLs: two in males and three in females (Table 2). However, subsequent analysis for interactive effects on aortic PWV reveals several interacting-loci affecting PWV that fulfilled the criteria for significant gene interaction (Fig. 2 and Table 3): AS-m1 (on chromosome 4) and $A S-m 2$ (on chromosome 16) in the male cohort (LOD 8.8, Table 3) and AS-f1 (on chromosome 9) and AS-f2 (on chromosome 11) in the female population (LOD 8.9, Table 3). Similarly, sex-specific genetic linkage analysis for strain did not identify significant QTLs. However, QTL analysis on the combined male and female populations detected a highly significant QTL (AS-1 on chromosome 3, LOD 4.3, Table 3) affecting aortic strain indicating that it influences aortic strain in a sex-independent manner.

Comparison of arterial stiffness and blood pressure QTLs We identified five QTLs influencing arterial stiffness in our F2 (Dahl S x R) intercross genetic analysis, four 

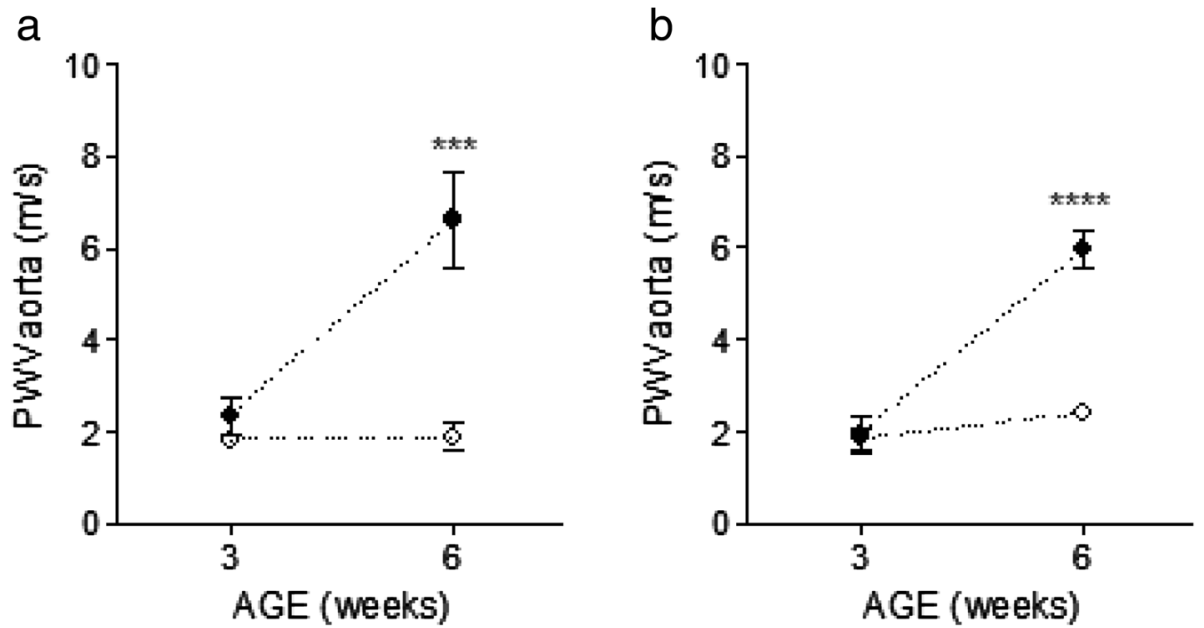

Fig. 1 Development of arterial stiffness in Dahl S and Dahl R rats measured by pulse wave velocity (PWV). Aortic PW (PW $V_{\text {aorta }}$ ) was measured in male (a) and female (b) Dahl S (black circle) and Dahl R (open circle) rats at 3 and 6 weeks of age. Dahl S males at 3 weeks of age, $n=4$; Dahl S males at 6 weeks of age, $n=4$; Dahl $\mathrm{S}$ females at 3 weeks of age, $n=5$; Dahl $\mathrm{S}$ females at 6 weeks of age, $n=6$; Dahl R males at 3 weeks of age, $n=4$, Dahl $\mathrm{R}$ males at 6 weeks of age, $n=6$; Dahl R females at 3 weeks of age, $n=6$, Dahl R females at 6 weeks of age, $n=6$. Values are means \pm s.e.m. ${ }^{* * *} P<0.001$, ${ }^{* * * *} P<0.00001$ (One Way ANOVA followed by Holm-Sidak Test for multiple comparisons)

affecting aortic PWV (on chromosomes 4, 16, 9 and 11) and one influencing aortic strain (on chromosome 3). Remarkably, none of the arterial stiffness QTLs co-localizes with previously reported blood pressure (BP) QTLs found in the same F2 (Dahl S x R) genetic intercrosses (Table 4). These data suggest the existence of distinct genetic determinants for salt-sensitive hypertension and arterial

Table 1 Aortic Pulse Wave Velocity and aortic strain in Dahl S, Dahl R and F2 intercross populations at six weeks of age

\begin{tabular}{llll}
\hline Cohort & $\mathrm{n}$ & $\mathrm{PWV}_{\text {aorta }}$ & STR $_{\text {aorta }}$ \\
\hline Males & 6 & $2.56 \pm 0.42$ & $0.179 \pm 0.012$ \\
Dahl R & 4 & $7.96 \pm 1.88$ & $0.142 \pm 0.008$ \\
Dahl S & 142 & $4.72 \pm 2.09$ & $0.176 \pm 0.002$ \\
F2 intercross & & & \\
Females & 6 & $2.51 \pm 0.92$ & $0.185 \pm 0.009$ \\
Dahl R & 6 & $6.42 \pm 0.54$ & $0.104 \pm 0.022$ \\
Dahl S & 140 & $4.77 \pm 1.95$ & $0.177 \pm 0.002$ \\
F2 intercross & & & \\
Statistical testing & & $P<0.001$ & $P=0.030$ \\
Dahl S vs Dahl R, M & & $P=0.004$ & $P=0.008$ \\
Dahl S vs F2, M & & $P=0.013$ & $P=n .5$. \\
Dahl R vs F2, M & & $P=0.001$ & $P<0.001$ \\
Dahl S vs Dahl R, F & & $P=0.040$ & $P<0.001$ \\
Dahl S vs F2, F & & $P=0.009$ & $P=n .5$. \\
Dahl R vs F2, F & &
\end{tabular}

Data is presented for populations and traits informative for QTL detection (male, female F2 populations phenotyped for PWV and STR). Values are Mean \pm standard deviation; PWV pulse wave velocity, STR aortic proximal strain, $M$ male, $F$ female; Animals were maintained on Purina $(0.4 \% \mathrm{NaCl})$ rat diet from inception; $P$, Two Way ANOVA followed by Holm-Sidak test for multiple comparisons stiffness, which is consistent with the temporal dissociation of onset of arterial stiffness and hypertension observed in Dahl salt-sensitive rats [6].

Although further QTL-gene mapping needs to be done, inspection of the rat chromosomal regions spanning the arterial stiffness QTLs reveals potential candidate genes based on vascular biology paradigms (Table 5). For QTL $A S-1$ on chromosome (Chr) 3 [40-60 Mbp], the Activin A receptor, Type 1 (ACVR1) at position 44.6 Mbp could underlie the $A S-1$ effect on aortic strain in our (Dahl $\mathrm{S} \times \mathrm{R}$ ) intercross. ACVR1 is a receptor for TGF- $\beta 1$, a multifunctional cytokine that influences the vascular wall via actions on endothelial cells and modulation of the extracellular matrix [9-14]. TGF- $\beta 1$ is a pro-fibrosis cytokine [11] and has been associated with fibrosclerotic development in

Table 2 QTLs with suggestive linkage for arterial stiffness in F2 (Dahl $\mathrm{S} \times \mathrm{R}$ )-intercross rats

\begin{tabular}{lllll}
\hline Chr & Reference marker & Location (Mbp) & LOD & Trait \\
\hline Males & & & & \\
5 & SNP2790733 & 55.43 & 2.3 & PW $_{\text {aorta }}$ \\
20 & SNP2803750 & 48.28 & 2.0 & PWW aorta \\
Females & & & & \\
9 & SNP2795465 & 30.75 & 2.8 & PW $_{\text {aorta }}$ \\
11 & SNP2797258 & 23.12 & 2.1 & PW $_{\text {aorta }}$ \\
15 & SNP2800195 & 50.59 & 2.1 & PW $_{\text {aorta }}$ \\
\hline
\end{tabular}

QTL quantitative trait locus, Chr chromosome, $P W V_{\text {aorta }}$ Pulse Wave Velocity in aorta, $L O D$ logarithm of the odds score derived from the likelihood ratio statistic using a factor of 4.6. For marker regression analysis significance was determined from 2000 permutations on data set: Highly significant LOD $\geq 4.3$, Significant $L O D \geq 3$.0and Suggestive linkage $L O D \geq 2.0$. SNP locations on rat genome as per NCBI Rat Genome Assembly Annotation Release 105 

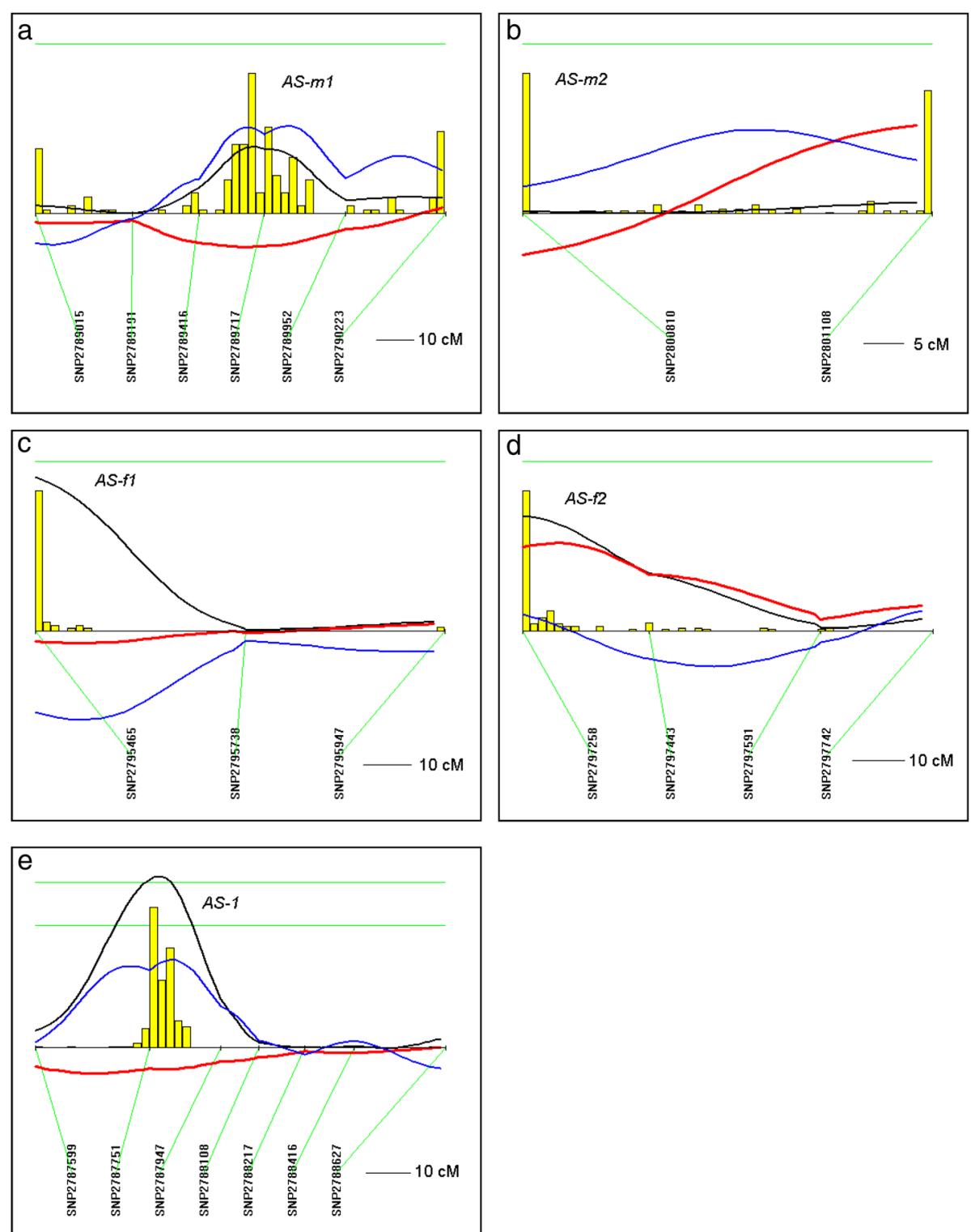

Fig. 2 QTLs for arterial stiffness (AS) in $F_{2}$ (Dahl $S \times R$ )-intercross rats. Chromosomes with interacting QTLs in male (a, b) and female (c, d) F2 cohorts and a chromosome with a highly significant QTL detected in the combined female + male F2 cohorts were analyzed by interval mapping with bootstrap resampling method to estimate a confidence interval (QTXb19 Map Manager): Panel a, chromosome 4 (AS-m1); b, chromosome 16 (AS-m2); c, chromosome 9 (AS-f1); d chromosome 11 (AS-f2) and e, chromosome 3 (AS-1). Yellow histograms represent the bootstrap-based confidence intervals for the detected QTLs. For a histogram with single peak, widths define the confidence interval for the QTL. Orientation of chromosomes: left $\rightarrow$ right starting from lowest Mbp. Horizontal green line for a-d mark LOD value for significant linkage (LOD $\geq 3.0)$. Horizontal green lines for panel e from top to bottom: highly significant $L O D \geq 4.3$; significant $L O D \geq 3.0$. Regression coefficient for additive effect (red lines); regression coefficient for dominance effect (blue lines); LOD (black lines)

varicose vessel walls [15]. Within the AS-f1 region on Chr. 9 (15-35 Mbp), the Vascular endothelial growth factor A (VEGF-A) gene resides at position 17.4 Mbp. VEGF-A is a valid candidate gene contributing to increased PWV since VEGF-A is increased in pulmonary microvascular endothelial cells subjected to increased flow pulsatility, a parameter associated with increased arterial stiffness in vivo [16]. Scanning of the AS-f2 QTL in the Chr. 11 (13-33 Mbp) region detects ADAMTS1 (A Disintegrin And Metalloproteinase $[\mathrm{ADAM}]$ with thrombospondin type 1 motif, 1) at coordinate 25.4 Mbp as candidate. ADAMTS1 has been implicated in arterial remodeling in multiple systems [17-19] and has been shown to be responsive to wall shear stress $[18,19]$.

$A S-f 1$ and $A S-f 2$ are female-specific interacting QTLs affecting aortic pulse wave velocity. The finding that 
Table 3 Arterial stiffness (AS) QTLs detected by marker regression and interaction analysis in F2 (Dahl S x R)-intercross rats

\begin{tabular}{lllllllllll}
\hline Chr & QTL & Locus A (Mbp) & Main A & Chr & QTL & Locus B (Mbp) & Main B & LOD T & LOD I & Trait (F2) \\
\hline 4 & AS-m1 & SNP2789717 (173.96) & 1.2 & 16 & AS-m2 & SNP2800810 (15.73) & 0.1 & 8.8 & 7.6 & PWV \\
9 & AS-f1 & SNP2795465 (30.75) & 2.8 & 11 & AS-f2 & SNP2797258 (23.12) & 2.1 & 8.9 & 4.0 & PWV \\
3 & AS-1 & SNP2787751 (50.64) & 4.3 & & & & & & STR $_{\text {aorta }}($ (M) + F)
\end{tabular}

QTL quantitative trait locus, Chr chromosome, PWV aorta Pulse Wave Velocity in aorta, STR aorta strain in aorta, M F2 male cohort, F F2 female cohort, LOD logarithm of the odds score derived from the likelihood ratio statistic using a factor of 4.6. For marker regression analysis significance was determined from 2000 permutations on data set: LOD 4.3 highly significant. Only interactive loci that exhibited a LOD $>8.0\left(P<10^{-5}\right)$ for the total effect and a LOD $>4.0(P<0.01)$ for the interactive effect are presented. Main A and Main B refer to the specific LOD for A and B main (single locus) effects respectively. LOD T, LOD for total effect; LOD I, LOD for the interaction. SNP locations on rat genome as per NCBI Rat Genome Assembly Annotation Release 105

ADAMTS1 inhibits endothelial cell proliferation by direct binding and sequestration of VEGF-A [20] is consistent with our hypothesis that ADAMTS1 and VEGF-A might underlie the interacting effect of $A S-f 1$ and $A S-f 2$ on aortic PWV. Although additional fine mapping studies are necessary to substantiate candidate genes within detected QTL regions influencing arterial stiffness, these candidate gene localizations in QTLs with significant

Table 4 QTLs for blood pressure and arterial stiffness in F2 (Dahl $S \times R$ ) intercross rats

\begin{tabular}{|c|c|c|c|c|}
\hline QTL & Trait & F2 cohort & Chr: location & Reference \\
\hline$A S-1$ & STR aorta & $M+F$ & Chr3: 40-60 Mbp & \\
\hline$A S-m 1$ & PWV & M & Chr4: 163-183 Mbp & \\
\hline AS-m2 & PWV aorta & M & Chr16: 6-26 Mbp & \\
\hline$B P-m 1$ & SBP & M & Chr1: 142-162 Mbp & [7] \\
\hline$B P-m 2$ & SBP & M & Chr1: 189-209 Mbp & [7] \\
\hline$B P-m 3$ & SBP & M & Chr20: 42-62 Mbp & [7] \\
\hline$B P-m 4$ & SBP & M & Chr2: 194-214 Mbp & [7] \\
\hline$B P-m 5$ & SBP & M & Chr11: 34-54 Mbp & [7] \\
\hline$A S-f 1$ & PWV aorta & $F$ & Chr9: 15-35 Mbp & \\
\hline$A S-f 2$ & PWV & $\mathrm{F}$ & Chr11: 13-33 Mbp & \\
\hline$B P-f 1$ & SBP & $\mathrm{F}$ & Chr5: 135-155 Mbp & [7] \\
\hline$B P-f 2$ & SBP & $F$ & Chr5: 103-123 Mbp & [7] \\
\hline$B P-f 3$ & SBP & $\mathrm{F}$ & Chr12: 4-24 Mbp & [7] \\
\hline BP-f4 & SBP & $\mathrm{F}$ & Chr2: 179-199 Mbp & [7] \\
\hline$B P-f 5$ & SBP & F & Chr2: 96-116 Mbp & [7] \\
\hline$B P-f 6$ & SBP & $\mathrm{F}$ & Chr1: 146-166 Mbp & [7] \\
\hline iXBP-f1 & SBP & $\mathrm{F}$ & Chr1: 189-209 Mbp & [7] \\
\hline$i x B P-f 2$ & SBP & $\mathrm{F}$ & Chr3: 115-135 Mbp & {$[7]$} \\
\hline ixBP-f3 & SBP & $\mathrm{F}$ & Chr14: 63-83 Mbp & [7] \\
\hline BP-pm 1 & SBP & $\mathrm{pmF}$ & Chr13: 73-93 Mbp & {$[8]$} \\
\hline BP-pm2 & SBP & $\mathrm{pmF}$ & Chr11: 53-73 Mbp & {$[8]$} \\
\hline BP-pm3 & DBP & $\mathrm{pmF}$ & Chr2: 254-270 Mbp & [8] \\
\hline BP-pm4 & DBP & $\mathrm{pmF}$ & Chr4: 164-184 Mbp & {$[8]$} \\
\hline BP-pm5 & SBP & $\mathrm{pmF}$ & Chr15: 18-38 Mbp & [8] \\
\hline
\end{tabular}

QTL quantitative trait locus, Chr chromosome, $P W V_{\text {aorta }}$ Pulse Wave Velocity in aorta, $S T R_{\text {aorta }}$ strain in aorta, $M$ F2 male cohort, $F$ F2 female cohort, pmF F2 post-menopausal female cohort, $B P$ blood pressure, $A S$ arterial stiffness, $M b p$ mega base pairs linkage integrate genetic factors with concordant pathophysiological paradigms of arterial stiffness.

Previous human genetic association studies have reported several candidate genes for loci associated with arterial stiffness (Table 6). However, only one out of ten loci was detected at a significant level with nine of them being suggestive for the association implying a degree of uncertainty in the association for these loci. Moreover, none of these candidate genes localize to syntenic regions harboring the detected rat arterial stiffness QTLs.

\section{Conclusions}

Altogether, our study demonstrates the involvement of distinct genetic determinants for aortic PWV and aortic strain in Dahl rats. In addition, based upon reported BP QTLs in equivalent intercrosses $[7,8]$ and our present findings, the data suggest the existence of distinct loci affecting BP and arterial stiffness in Dahl salt-sensitive hypertensive rats. Moreover, as found with increasing number of traits [7, 21-25], arterial stiffness portrays sex-specificity implying the existence of differential genetic mechanisms underlying vessel stiffening in male and female Dahl salt-sensitive rats. The subsequent identification of genes underlying the aortic pulse wave velocity and strain QTLs will define the genetic basis of aortic stiffness in the Dahl salt-sensitive hypertensive rat model. This will provide a critical first step towards the elucidation of novel aortic-stiffness genetic mechanisms, and provides the basis for further study of aortic stiffness genes as putative genetic biomarkers of adult-onset hypertension and its target-organ complications.

\section{Methods}

This study was performed in strict accordance with the recommendations in the Guide for the Care and Use of Laboratory Animals of the National Institutes of Health. The protocol was approved by the Committee on the Ethics of Animal Experiments of Boston University School of Medicine (Permit Number: AN-14966). All measurements were performed under isoflurane anesthesia, and every effort was made to minimize suffering. Euthanasia was done by exsanguination and removal of vital tissue under anesthesia. Secondary euthanasia procedure of pneumothorax was also done under anesthesia (isoflurane 
Table 5 Candidate genes for arterial stiffness QTLs detected with significant linkage in F2 (Dahl S x R) rat intercross

\begin{tabular}{llllll}
\hline QTL & QTL-location & Trait & Symbol & Location & Description \\
\hline AS-1 & Chr3: 40-60 Mbp & Strain & ACVR1 & 44.6 Mbp & Activin A receptor, Type 1 \\
AS-m1 & Chr4: 163-183 Mbp & PW & & & \\
AS-m2 & Chr16: 6-26 Mbp & PWV & & & \\
AS-f1 & Chr9: 15-35 Mbp & PWV & VEGF A & $17.4 \mathrm{Mbp}$ & Vascular endothelial growth factor A \\
AS-f2 & Chr11: 13-33 Mbp & PWV & ADAMTS 1 & 25.4 Mbp & A Disintegrin And Metalloproteinase (ADAM) with thrombospondin type 1 motif, 1
\end{tabular}

QTL quantitative trait locus, [AS-m1]-[AS-m2] are male-specific interacting QTLs, [AS-f1]-[AS-f2] are female-specific interacting QTLs, Chr chromosome, Mbp mega base pairs; Strain, aortic strain, PWV aortic pulse wave velocity. QTL and gene locations on rat genome as per NCBI Rat Genome Assembly Annotation Release 105

or ketamine/xylazine at $40-80 \mathrm{mg} / \mathrm{kg}$ ketamine + 5$20 \mathrm{mg} / \mathrm{kg}$ xylazine). This method is consistent with the recommendation of the Panel of Euthanasia of the American Veterinary Medical Association (AVMA http://grants.nih.gov/grants/guide/notice-files/NOT-OD-01-029.html).

\section{Genetic crosses}

Inbred Dahl S/jrHsd and Dahl R/jrHsd rats were obtained from Harlan (Indianapolis, Indiana). Parental strains (Dahl $\mathrm{R} / \mathrm{jrHsd} \times$ Dahl S/jrHsd) were crossed to produce F1 progeny. The F2 subjects were derived from brother-to-sister mating of F1 hybrids to produce the F2 female $(n=140)$ and F2 male $(n=142)$ segregating populations. Animals were maintained on Purina $5001(0.4 \% \mathrm{NaCl})$ rat diet from inception.

\section{Arterial stiffness measurements}

We performed high-resolution ultrasonography measurement of pulse wave velocity (PWV) in rat abdominal aorta (average distance $=1.0 \mathrm{~cm}$ ) essentially as described [6] . PWV was defined as $\Delta \mathrm{d} / \Delta \mathrm{t}$, distance (d) between two points divided by the difference in transit time $(t)$ of the pressure wave arrival at said two points. The transit time was defined as the time from the peak of the ECG R-wave to the foot of the velocity upstroke [26], with the foot defined as the point at the end of diastole when the steep rise of the wave front begins [27]. Measurement of strain $\left(\mathrm{D}^{\mathrm{SYS}}-\mathrm{D}^{\mathrm{DIAS}}\right) / \mathrm{D}^{\mathrm{DIAS}}$, where $\mathrm{D}=$ diameter of artery, was also done in abdominal aorta as another measure of arterial stiffness and distensibility. Each measurement of PWV and strain represents the average of five independent determinations per subject. We characterized 12 Dahl R, 10 Dahl S, 142 F2 males and 140 F2 females at six weeks of age. We also measured PWV in 10 Dahl $R$ and 9 Dahl S rats at three weeks of age. Animals were not phenotyped for blood pressure. The ultrasound micro-imaging and Doppler ultrasound studies were done with $0.8 \%$ isoflurane anesthesia - hence expected to not induce non-physiological artifacts since it is less than the $1 \%$ isoflurane anesthesia level defined as "baseline level" for coronary blood flow with heart rates similar to sleeping mice [28] and documented to not alter aortic impedance [29].

\section{Intercross linkage analysis}

Phenotype distributions were analyzed for normality; data transformations were done when necessary and datasets that passed Kolmogorov-Smirnov normality testing (SigmaPlot 11.2) were used for linkage analysis. Specifically, the current QTL analysis was performed

Table 6 Candidate genes for loci associated with arterial stiffness (cfPWV) in humans

\begin{tabular}{llllll}
\hline Cohort & Sig & H-Position & Symbol & Description & Rat Position \\
\hline Framingham & Suggestive & Chr15: $90.9 \mathrm{Mbp}$ & FURIN & Paired basic amino acid cleaving enzyme & Chr1: 142.2 Mbp \\
Framingham & Suggestive & Chr15: $98.6 \mathrm{Mbp}$ & IGF1R & Insulin-like growth factor-1 receptor & Chr1: 128.9 Mbp \\
Framingham & Suggestive & Chr15: $99.6 \mathrm{Mbp}$ & MEF2A & Myocyte-specific enhancer factor 2A & Chr1: 128.4 Mbp \\
Framingham & Suggestive & Chr15: $101.2 \mathrm{Mbp}$ & CHSY1 & Chondroitin synthase 1 & Chr1: 127.0 Mbp \\
Framingham & Suggestive & Chr15: $101.3 \mathrm{Mbp}$ & PACE4 & Proprotein convertase subtilisin/kexin type 6 & Chr1: 126.7 Mbp \\
Framingham & Suggestive & Chr2: $70.7 \mathrm{Mbp}$ & ADD2 & B-adducin & Chr4: 117.7 Mbp \\
Framingham & Suggestive & Chr2: $75.0 \mathrm{Mbp}$ & TACR1 & Neurokinin-1 receptor & Chr4: 113.2 Mbp \\
Framingham & Suggestive & Chr2: $96.1 \mathrm{Mbp}$ & ADRA2B & a-2B adrenergic receptor & Chr3: 119.8 Mbp \\
Framingham & Suggestive & Chr7: $22.7 \mathrm{Mbp}$ & IL6 & Interleukin-6 & Chr4: 3.0 Mbp \\
Europe & Significant & Chr14: $99.2 \mathrm{Mbp}$ & 3'-BCL11B gene desert & 3'-B-cell CLL/lymphoma 11B (zinc finger protein) & Chr6: 131.9 Mbp \\
& & & & gene desert &
\end{tabular}

cfPWV carotid-femoral pulse wave velocity, Sig significance, Chr chromosome, Mbp mega base pairs, H-Position human position as per NCBI Human Genome Assembly Annotation Release 106; Rat position as per NCBI Rat Genome Assembly Annotation Release 105. Framingham cohort (1480 participants) as reported by [2]; European cohort (20634 participants) as reported by [32] 
using Log 10 [aortic PWV] and untransformed aortic Strain as quantitative traits. Data analysis was not controlled for BP since at six weeks of age Dahl S and Dahl $R$ rats have equivalent BP [30], therefore not likely to be an independent confounder of the arterial stiffness measurements. Linkage maps, marker regression and composite interval mapping were done with the Map Manager QTXb19 (MMQTXb19) program for windows [31] which generates a likelihood ratio statistic (LRS) as a measure of the significance of a possible QTL. Genetic distances were calculated using Kosambi mapping function (genetic distances are expressed in centiMorgan, cM). Critical significance values (LRS values) for interval mapping were determined by a permutation test (2000 permutations at all loci tested) on our male and female cohorts using Kosambi mapping function and a dominant, recessive or additive regression model. Values for significant linkage LRS $=13.8$ (LOD 3.0) and for highly significant linkage LRS $=19.8$ (LOD 4.3). LRS 4.6 delineates LOD 1-support interval. Confidence interval for a QTL location was estimated by bootstrap resampling method wherein histogram single peak delineates the QTL and peak widths define confidence interval for the QTL. We also performed interaction analysis using the Map Manager QTXb19 program applying a two-stage test paradigm for determination of interaction in which the pair of loci must pass two tests in order to be reported as having a significant interaction effect. First, the significance of the total effect of the two loci must be $<0.00001$ and second, the pairs of loci must exhibit a $P$ value $<0.01$ for the interaction effect.

\section{Genotyping}

DNA purification from spleen tissue samples was done by using the DNeasy Blood \& Tissue Kit (QIAGEN) following manufacturer's instructions. SNP genotyping was carried out on an Applied Biosystems 7900 Real-Time PCR System. SNPs $(n=98)$ and SSLP markers $(n=12)$ were identified as informative markers between our parental strains by using SNPlotyper from the Rat Genome Database. SNP assays (TaqMan assays) were procured from Applied Biosystems and were validated in our laboratory.

\section{Statistical analyses}

We performed one-way ANOVA followed by all pairwise multiple comparisons using the Holm-Sidak Test for PWV and Strain measurements as indicated per experimental comparison.

\section{Abbreviations}

Dahl S: Dahl salt-sensitive; Dahl R: Dahl salt-resistant; QTL: quantitative trait locus; LOD: logarithm of the odds score; PWV: pulse wave velocity;

CYP11B2: cytochrome P450, family 11, subfamily B, polypeptide 2; NOS3: nitric oxide synthase 3 (endothelial cell); AS: arterial stiffness; BP: blood pressure; ACVR1: Activin A receptor, Type 1; VEGF-A: Vascular endothelial growth factor A; ADAMTS1: A Disintegrin And Metalloproteinase with thrombospondin type 1 motif, 1; SNP: single nucleotide polymorphism; SSLP: single strand length polymorphism; Chr: chromosome; M: male; F: female; pmF: post-menopausal female; Mbp: mega base pairs; cfPWV: carotid-femoral pulse wave velocity; FURIN: paired basic amino acid cleaving enzyme; IGF1R: insulin-like growth factor-1 receptor; MEF2A: myocyte-specific enhancer factor 2A;

CHSY1: chondroitin synthase 1; PACE4: proprotein convertase subtilisin/kexin type 6; ADD2: $\beta$-adducin; TACR1: neurokinin-1 receptor; ADRA2B: $a-2 B$ adrenergic receptor; IL6: interleukin-6; 3'-BCL11B: 3'-B-cell CLL/lymphoma 11B (zinc finger protein).

\section{Competing interests}

The authors declare that they have no competing interests.

\section{Authors' contributions}

NR-O designed the experiments, supervised the study, carried out the linkage and QTL analysis and drafted the manuscript, VLH contributed to write the manuscript and help in the analysis of the different arterial stiffness measurements, JLD performed all ultrasound measurements on parental strains and male and half of the female F2 intercross population, NB and NJG performed the ultrasound measurements on the remaining half of the female F2 population, KAP performed all intercrosses to generate both male and female F2 segregating populations and performed all the genotyping. All authors have read and approved the final version of the manuscript.

\section{Acknowledgements}

Research described in this article was supported by National Heart, Lung, and Blood Institute Grant HL105286, awarded to N. Ruiz-Opazo.

Received: 9 September 2015 Accepted: 22 December 2015

Published online: 11 January 2016

\section{References}

1. Ge D, Young TW, Wang X, Kapuku GK, Treiber FA, Snieder H. Heritability of arterial stiffness in black and white American youth and young adults. Am J Hypertens. 2007;20:1065-72.

2. Mitchell GF, DeStefano AL, Larson MG, Benjamin EJ, Chen MH, Vasan RS, et al. Heritability and a genome-wide linkage scan for arterial stiffness, wave reflection, and mean arterial pressure. The Framingham Heart Study. Circulation. 2005;112:194-9.

3. Lacolley P, Challande P, Osborne-Pellegrin M, Regnault V. Genetics and pathophysiology of arterial stiffness. Cardiovasc Res. 2009;81:637-48.

4. Gaugier D, Behmoaras J, Argoud K, Wilder SP, Pradines C, Bihoreau MT, et al. Chromosomal mapping of QTL controlling elastin content in rat aorta. Hypertension. 2005;45:460-6.

5. Behmoaras J, Osborne-Pellegrin M, Gauguier D, Jacob MP. Characteristics of the aortic elastic network and related phenotypes in seven inbred rat strains. Am J Physiol Heart Circ Physiol. 2005;288:H769-H77.

6. Herrera VL, Decano JL, Giordano N, Moran AM, Ruiz-Opazo N. Aortic and carotid arterial stiffness and epigenetic regulator gene expression changes precede blood pressure rise in stroke-prone Dahl salt-sensitive hypertensive rats. PLoS One. 2014;9(9):e107888.

7. Herrera VL, Tsikoudakis A, Ponce LR, Matsubara Y, Ruiz-Opazo N. Sex-specific QTLs and interacting-loci underlie salt-sensitive hypertension and target-organ complications in Dahl S/jr HS hypertensive rats. Physiol Genomics. 2006;26:172-9.

8. Herrera VL, Pasion KA, Moran AM, Ruiz-Opazo N. Differential genetic basis for pre-menopausal and post-menopausal salt-sensitive hypertension. PLoS One. 2012;7(8):e43160.

9. Border WA, Noble NA. Transforming growth factor beta in tissue fibrosis. New Engl J Med. 1994;331:1286-92.

10. Robertson AK, Rudling M, Zhou X, Gorelik L, Flavell RA, Hansson GK Disruption of TGF-beta signaling in $T$ cells accelerates atherosclerosis. J Clin Invest. 2003;112:1342-50.

11. Mallat Z, Tedgui A. The role of transforming growth factor beta in atherosclerosis: novel insights and future perspectives. Curr Opin Lipidol. 2002;13:523-9.

12. Roberts AB, Sporn MB, Assoian RK, Smith JM, Roche NS, Wakefield LM, et al. Transforming growth factor type beta: rapid induction of fibrosis and 
angiogenesis in vivo and stimulation of collagen formation in vitro. Proc Natl Acad Sci U S A. 1986;83:4167-71.

13. Grainger DJ, Metcalfe JC. A pivotal role for TGF-beta in atherogenesis? Biol Rev Camb Philos Soc. 1995;70:571-96.

14. Topper JN. TGF-beta in the cardiovascular system: molecular mechanisms of a context-specific growth factor. Trends Cardiovasc Med. 2000;10:132-7.

15. Jacob T, Hingorani A, Ascher E. Overexpression of transforming growth factor-betal correlates with increased synthesis of nitric oxide synthase in varicose veins. J Vasc Surg. 2005;41:523-30.

16. Li M, Scott DE, Shandas R, Stenmark KR, Tan W. High pulsatility flow induces adhesion molecule and cytokine mRNA expression in distal pulmonary artery endothelial cells. Ann Biomed Eng. 2009;37:1082-92.

17. Jonsson-Rylander AC, Nilsson T, Fritsche-Danielson R, Hammarstrom A, Behrendt M, Andersson JO, et al. Role of ADAMTS-1 in atherosclerosis: remodeling of carotid artery, immunohistochemistry, and proteolysis of versican. Arterioscler Thromb Vasc Biol. 2005;25:180-5.

18. Dolan JM, Sim FJ, Meng H, Kolega J. Endothelial cells express a unique transcriptional profile under very high wall shear stress known to induce expansive arterial remodeling. Am J Physiol Cell Physiol. 2012;302:C1109-C18.

19. Dolan JM, Meng H, Sim FJ, Kolega J. Differential gene expression by endothelial cells under positive and negative streamwise gradients of high wall shear stress. Am J Physiol Cell Physiol. 2013;305:C854-C66.

20. Luque A, Carpizo DR, Iruela-Arispe ML. ADAMTS1/METH1 inhibits endothelial cell proliferation by direct binding and sequestration of $\mathrm{VEGF}_{165}$. J Biol Chem. 2003;278:23656-65.

21. Glorioso N, Herrera VL, Bagamasbad P, Filigheddu F, Troffa C, Argiolas G, et al. Association of ATP1A1 and Dear SNP-haplotypes with essential hypertension: sex-specific and haplotype-specific effects. Circ Res. 2007;100:1522-9.

22. Herrera VL, Decano JL, Bagamasbad P, Kufahl T, Steffen M, Ruiz-Opazo N. Sex-specific hippocampus-dependent cognitive deficits and increased neuronal autophagy in DEspR haploinsufficiency in mice. Physiol Genomics. 2008:35:316-29.

23. Herrera VL, Bagamasbad P, Decano JL, Ruiz-Opazo N. AVR/NAVR deficiency lowers blood pressure and differentially affects urinary concentrating ability, cognition and anxiety-like behavior in male and female mice. Physiol Genomics. 2011;43:32-42.

24. Herrera VL, Pasion KA, Tan GA, Moran AM, Ruiz-Opazo N. Sex-specific effects on spatial learning and memory, and sex-independent effects on blood pressure of a $<3.3 \mathrm{Mbp}$ rat chromosome 2 QTL region in Dahl salt-sensitive rats. PLoS One. 2013;8(7):e67673.

25. Glorioso N, Herrera VL, Didishvili T, Ortu MF, Zaninello R, Fresu G, et al. Sexspecific effects of NLRP6/AVR and ADM loci on susceptibility to essential hypertension in a Sardinian population. PLoS One. 2013;8(10):e77562.

26. Williams R, Needles A, Cherin E, Zhou YQ, Henkelman RM, Adamson SL, et al. Noninvasive ultrasonic measurement of regional and local pulse-wave velocity in mice. Ultrasound Med Biol. 2007:33:1368-75.

27. Zhang $X$, Greenleaf JF. Noninvasive generation and measurement of propagating waves in arterial walls. J Acoust Soc Am. 2006;119:1238-43.

28. Hartley CJ, Reddy AK, Madala S, Michael LH, Entman ML, Taffet GE. Doppler estimation of reduced coronary flow reserve in mice with pressure overload cardiac hypertrophy. Ultrasound Med Biol. 2008;34:892-901.

29. Reddy AK, Namiranian K, Looyd EE, Bryan RM, Taffet GE, Hartley CJ. Effect of isoflurane on aortic impedance in mice. Conf Proc IEEE Eng Med Biol Soc. 2009:1:1104-5.

30. Kong JQ, Taylor DA, Fleming WW. Sustained hypertension in Dahl rats. Negative correlation of agonist response to blood pressure. Hypertension. 1995;1:139-45.

31. Manly KF, Cudmore Jr RH, Meer JM. Map Manager QTX, cross-platform software for genetic mapping. Mamm Genome. 2001;12:930-2.

32. Mitchell GF, Verwoert GC, Tarasov KV, Isaacs A, Smith AV, Yasmin, et al. Common genetic variation in the $3^{\prime}-B C L 11 B$ gene desert is associated with carotid-femoral pulse wave velocity and excess cardiovascular disease risk. The AortaGen Consortium. Circ Cardiovasc Genet. 2012;5:81-90,

\section{Submit your next manuscript to BioMed Central and we will help you at every step:}

- We accept pre-submission inquiries

- Our selector tool helps you to find the most relevant journal

- We provide round the clock customer support

- Convenient online submission

- Thorough peer review

- Inclusion in PubMed and all major indexing services

- Maximum visibility for your research

Submit your manuscript at www.biomedcentral.com/submit
Biomed Central 